Pacific

Journal of

Mathematics

CONVEX EIGENFUNCTION OF A DRIFTING LAPLACIAN OPERATOR AND THE FUNDAMENTAL GAP

LI MA AND BAIYU LIU 


\title{
CONVEX EIGENFUNCTION OF A DRIFTING LAPLACIAN OPERATOR AND THE FUNDAMENTAL GAP
}

\author{
LI MA AND BAIYU LIU
}

\begin{abstract}
We study the convexity of the first eigenfunction of the drifting Laplacian operator with zero Dirichlet boundary value provided a suitable assumption to the drifting term is added. We firstly generalize some results of N. Korevaar and S.-T. Yau to gain a Hessian estimate of the first eigenfunction. As an application, we use this Hessian estimate to get a lower bound of the difference of the first and second eigenvalues of the drifting Laplacian. At the end we also find a lower bound when the Hessian estimate does not hold.
\end{abstract}

\section{Introduction}

It is a significant problem in mathematical physics and differential geometry to study the eigenvalue estimates of self-adjoint operators in Hilbert spaces [ $\mathrm{Li}$ and Yau 1986; Schoen and Yau 1994; Li and Wang 2005; Ma and Zhu 2007]. Given a smooth convex bounded domain $\Omega \subset \mathbb{R}^{n}$, we consider the Dirichlet eigenvalue problem

$$
\begin{cases}-\Delta_{h} f+V f=\lambda f, & \text { in } \Omega \\ f=0, & \text { on } \partial \Omega,\end{cases}
$$

where $\Delta_{h}=\Delta-\nabla h \cdot \nabla$ and $h, V$ are two given smooth functions on the closure of $\Omega$. In the $h=0$ case, $\Delta_{0}$ is the standard Laplacian operator in $\mathbb{R}^{n}$ such that $\Delta u=u^{\prime \prime}$ when $n=1$. See [Da Prato and Lunardi 2004] for interesting results with the drifting Laplacian operator. There are very few results on the eigenvalue estimates for the problem (1) — see [González and Negrin 1999] — and we only find some related interesting results in [Kawohl 1985; Ni 2004; Setti 1993].

Throughout this paper, we shall use the following basic properties of the operator $-\Delta_{h}+V$ :

Property 1. The first and second eigenvalues $\lambda_{1}$ and $\lambda_{2}$ of the operator $-\Delta_{h}+V$ satisfy $0<\lambda_{1}<\lambda_{2}$.

MSC2000: primary 35P15; secondary 35P15.

Keywords: eigenvalue, drifting Laplacian, Hessian estimate, fundamental gap.

The research is partially supported by the National Natural Science Foundation of China 10631020 and SRFDP 20060003002. 
Property 2. The first and second eigenfunctions $f_{1}$ and $f_{2}$ are both smooth on $\bar{\Omega}$. Moreover, $f_{1}>0$.

Our overall plan is first to investigate the convexity of the first eigenfunction of problem (1), by enhancing some results of N. Korevaar [1983]. Then we use the convexity properties to extend results of S.-T. Yau [2003] (where $h=0$ ) to the problem (1).

In the case when $h=0$, one of these results is that for a convex domain $\Omega$ with a potential $V$, if the Hessian of $V$ has a positive lower bound, then the first eigenfunction of the operator $-\Delta+V$ is Log concave. In our case when the drifting term is added, we will show that if the Hessian of

$$
\psi:=V-\frac{1}{2} \Delta h+\frac{1}{4}|\nabla h|^{2}
$$

has a positive lower bound, then the first eigenfunction of the operator $-\Delta_{h}+V$ is Log concave compared with the drifting term $h$. To be precise:

Theorem 1. Let $\Omega$ be a smooth convex bounded domain in $\mathbb{R}^{n}$. Suppose

$$
\operatorname{Hess}(\psi)-c I \geq 0
$$

with some constant $c>0$. Then we have

$$
\operatorname{Hess}\left(\frac{h}{2}+\varphi\right)-\sqrt{\frac{c}{2}} I \geq 0
$$

where $\varphi=-\log f_{1}$.

Remark. When $V=0$, the function $\psi=-\frac{1}{2} \Delta h+\frac{1}{4}|\nabla h|^{2}$ has a geometric meaning; see [Ma and Liu 2008].

After applying Theorem 1, we deduce the following corollary by using Theorem 1.1 in [Yau 2003].

Corollary 2. Let $\Omega$ be a smooth convex bounded domain in $\mathbb{R}^{n}$. Suppose

$$
\operatorname{Hess}(\psi)-c I \geq 0
$$

with some constant $c>0$. Then

$$
\lambda_{2}-\lambda_{1} \geq \frac{\theta^{2}(\beta)}{\operatorname{diam}(\Omega)^{2}}+\beta \sqrt{c},
$$

where $\theta(\beta)=\arcsin (1 / \sqrt{1+\beta /(\sqrt{2}-\beta)})$ and $0<\beta<\sqrt{2}$.

Even when $\psi$ is not convex, we can find an estimate of the fundamental gap of $-\Delta_{h}+V$ by using the following gradient estimate for function $u=f_{2} / f_{1}$, where $f_{1}$ and $f_{2}$ are the first and second eigenfunctions of $-\Delta_{h}+V$. Actually, we follow the methods of S.-T. Yau [2003]. Since our results are more general than his results, we shall give complete proofs. 
Theorem 3. Let $\Omega$ be a smooth convex bounded domain in $\mathbb{R}^{n}$. Let $\kappa_{i}(x)(1 \leq i \leq n)$ be the eigenvalues of $\operatorname{Hess}(h / 2+\varphi)$ at $x$, and let $\lambda=\lambda_{2}-\lambda_{1}$. For any $\varepsilon>0$, let

$$
\alpha=2 \lambda\left(1+\varepsilon^{-1}\right)-4 \min _{1 \leq i \leq n} \inf _{x \in \Omega} \kappa_{i} .
$$

Assume that

$$
\min _{1 \leq i \leq n} \inf _{x \in \Omega} \kappa_{i}(x) \leq 0 .
$$

Then we have the following estimate for the gradient of $u=f_{2} / f_{1}$ :

$$
\frac{|\nabla u|}{c-u} \leq \sqrt{\alpha}(\log c-\log (c-u))^{1 / 2},
$$

where $c=(1+\varepsilon) \sup _{x \in \Omega} u$.

After using this gradient estimate, we can derive a lower bound for the difference of eigenvalues $\lambda$.

Corollary 4. Let $\Omega$ be a smooth convex bounded domain in $\mathbb{R}^{n}$. Suppose

$$
\min _{1 \leq i \leq n} \inf _{x} \kappa_{i} \geq-a, \quad a \geq 0 .
$$

Then the fundamental gap of the operator $-\Delta_{h}+V$ satisfies

$$
\lambda_{2}-\lambda_{1} \geq 2(\operatorname{diam} \Omega)^{-2} \exp \left(-a(\operatorname{diam} \Omega)^{2}-1\right) .
$$

We point out that the constant $e^{-1}$ in [Yau 2003, (3.15)] is missing.

Remark. Because a convex domain can be approximated by strictly convex domains, we shall prove the results only for strictly convex domains. In the following we assume that $\Omega$ is a smooth strictly convex bounded domain in $\mathbb{R}^{n}$.

\section{Preliminary results}

By Property $2, f_{1}$ is a positive function. Then $u=f_{2} / f_{1}$ is a well-defined smooth function in $\Omega$. We firstly try to find the equation it satisfies. Recall that $\lambda=\lambda_{2}-\lambda_{1}$.

Lemma 5. $\Delta_{h} u=-\lambda u-2 \nabla u \cdot \nabla \log f_{1}$.

Proof. By direct computation, we have

$\Delta u$

$$
\begin{aligned}
& =\frac{\Delta f_{2}}{f_{1}}-2 \frac{\nabla f_{1} \cdot \nabla f_{2}}{f_{1}^{2}}-\frac{f_{2}}{f_{1}^{2}} \Delta f_{1}+2 \frac{f_{2}}{f_{1}^{3}}\left|\nabla f_{1}\right|^{2} \\
& =\frac{1}{f_{1}^{2}}\left(-\lambda_{2} f_{1} f_{2}+\lambda_{1} f_{1} f_{2}\right)+\frac{1}{f_{1}^{2}}\left(f_{1} \nabla h \cdot \nabla f_{2}-f_{2} \nabla h \cdot \nabla f_{1}\right)-2 \frac{\nabla f_{1} \cdot \nabla f_{2}}{f_{1}^{2}}+2 f_{2} \frac{\left|\nabla f_{1}\right|^{2}}{f_{1}^{3}} \\
& =-\lambda u+\frac{\nabla h \cdot \nabla f_{2}}{f_{1}}-\frac{f_{2}}{f_{1}^{2}} \nabla h \cdot \nabla f_{1}-2 \frac{\nabla f_{1} \cdot \nabla f_{2}}{f_{1}^{2}}+2 f_{2} \frac{\left|\nabla f_{1}\right|^{2}}{f_{1}^{3}} .
\end{aligned}
$$


Now, taking into account the relations

$$
\nabla u \cdot \nabla \log f_{1}=\frac{\nabla f_{1} \cdot \nabla f_{2}}{f_{1}^{2}}-f_{2} \frac{\left|\nabla f_{1}\right|^{2}}{f_{1}^{3}}, \quad \nabla h \cdot \nabla u=\frac{\nabla h \cdot \nabla f_{2}}{f_{1}}-\frac{f_{2}}{f_{1}^{2}} \nabla h \cdot \nabla f_{1},
$$

we obtain

$$
\Delta u=-\lambda u+\nabla h \cdot \nabla u-2 \nabla u \cdot \nabla \log f_{1},
$$

which proves the lemma.

We now consider the smoothness of the function $u$ up to the boundary. This is a standard matter, but for the sake of completeness we include it here.

Lemma 6. Let $\Omega \subset \mathbb{R}^{n}$ be a smooth bounded domain. Then $u=f_{2} / f_{1}$ is smooth up to the boundary $\partial \Omega$. Moreover, it satisfies the Neumann condition on the boundary.

Proof. For all $p \in \partial \Omega$, let us choose local coordinates $\left\{x_{1}, x_{2}, \ldots, x_{n}\right\}$ on a sufficiently small neighborhood $U$ such that $p \in U \cap \partial \Omega=U \cap\left\{x_{1}=0\right\}$.

Since

$$
\left\{\begin{array}{l}
f_{1}=0 \text { on } \partial \Omega \\
f_{1}>0 \text { in } \Omega
\end{array}\right.
$$

by the Hopf lemma we have $\partial f_{1} / \partial x_{1} \neq 0$ on $\partial \Omega$. Furthermore, $f_{1}$ is smooth up to the boundary, thus one can consider $f_{1}$ as a smooth function which is defined on $U$ restricted to $U \cap \bar{\Omega}$. Using the Malgrange preparation theorem [Schoen and Yau 1994], we have locally

$$
f_{1}=g_{1} \cdot x_{1}, x \in \bar{\Omega} \cap U,
$$

where $g_{1}$ satisfies $g_{1} \neq 0$ and is smooth on $\bar{\Omega} \cap U$. Moreover, $f_{2}$ is identically zero on $\partial \Omega$. Applying the Malgrange preparation theorem again, we can write locally

$$
f_{2}=g_{2} \cdot x_{1},
$$

where $g_{2}$ is also a smooth function on $\bar{\Omega} \cap U$. It is an immediate consequence that

$$
u=\frac{f_{2}}{f_{1}}=\frac{g_{2}}{g_{1}}
$$

must be smooth on $\overline{\boldsymbol{\Omega}} \cap U$. Therefore, $u$ is smooth up to the boundary $\partial \boldsymbol{\Omega}$.

By using Equation (5), we have

$$
2 \nabla u \cdot \nabla \log f_{1}=-\Delta u-\lambda u+\nabla h \cdot \nabla u .
$$


Since $h$ is smooth up to the boundary, as we have assumed, $\Delta u, \nabla h \cdot \nabla u$ and $u$ are all smooth up to the boundary and thus attain finite values on $\partial \Omega$. Therefore,

$$
\nabla u \cdot \nabla \log f_{1}=\frac{1}{f_{1}} u_{1}\left(f_{1}\right)_{1}+\frac{1}{f_{1}} \sum_{i=2}^{n} u_{i}\left(f_{1}\right)_{i}
$$

achieves finite value on $\partial \Omega$ as well. Multiply both sides of Equation (7) by $f_{1}$. A simple computation shows

$$
f_{1}\left(\nabla u \cdot \nabla \log f_{1}\right)-\sum_{i=2}^{n} u_{i}\left(f_{1}\right)_{i}=u_{1}\left(f_{1}\right)_{1}
$$

From the fact that $f_{1}=0$ on $\partial \Omega$, we have $\left(f_{1}\right)_{i}=0$ on $\partial \Omega$ for $i \in\{2,3, \ldots, n\}$. Thus we see that the left-hand side of (8) tends to 0 as $x$ tends to $p \in \partial \Omega$. Therefore,

$$
\lim _{x \rightarrow p} u_{1}\left(f_{1}\right)_{1}=0 .
$$

Nevertheless, since $\left(f_{1}\right)_{1} \neq 0$ on $\partial \Omega$, we get the important observation:

$$
u_{1}(p)=0, p \in \partial \Omega \text {. }
$$

Thus we get $\partial u / \partial v=0$ on $\partial \Omega$, where $v$ is the outward normal vector to $\partial \Omega$. That is to say $u$ satisfies the Neumann condition on the boundary $\partial \Omega$.

Let us compare (5) with (9) carefully. If $h / 2-\log f_{1}$ is strictly convex, then we can gain a lower bounded of $\lambda=\lambda_{2}-\lambda_{1}$ by applying the following lemma, obtained by S.-T. Yau [2003].

Lemma 7. Suppose the Ricci curvature of $\Omega$ is nonnegative and $\partial \Omega$ is convex. Let the function $u$ be a solution of the problem

$$
\left\{\begin{array}{l}
\Delta u=-\left(\lambda_{2}-\lambda_{1}\right) u+2 W \cdot \nabla u \\
\frac{\partial u}{\partial v}=0
\end{array}\right.
$$

where $W$ is a vector field such that $W_{i, i} \geq \sqrt{c / 2}>0$. Then

$$
\lambda_{2}-\lambda_{1} \geq \frac{\theta^{2}(\beta)}{(\operatorname{diam} \Omega)^{2}}+\beta \sqrt{c},
$$

where $\beta$ is any number in $(0, \sqrt{2})$ and $\theta(\beta)=\arcsin \left(1+\frac{\beta}{\sqrt{2}-\beta}\right)^{-1 / 2}$.

Proof. This is Theorem 1.1 in [Yau 2003].

To find the condition under which $h / 2-\log f_{1}$ can be strictly convex, we will introduce the concavity function $\mathscr{b}$ and after that we will introduce two maximum principles for it. 
Definition 8. Suppose $u$ is defined on the closure of a bounded domain $\Omega$. The function

$$
\mathscr{C}\left(y_{1}, y_{3}, \mu\right)=u\left(y_{2}\right)-\mu u\left(y_{3}\right)-(1-\mu) u\left(y_{1}\right),
$$

defined for $y_{1}, y_{3} \in \bar{\Omega}$ such that $y_{2}=\mu y_{3}+(1-\mu) y_{2} \in \bar{\Omega}, 0 \leq \mu \leq 1$, is called the concavity function of $u$.

This function was introduced in [Korevaar 1983]. It is used to measure how much a function $u$ fails to be convex. We can see that the function $u$ is convex if and only if $\mathscr{C} \leq 0$ for all $y_{1}, y_{2}, y_{3}$ as above.

Notice that $\mathscr{C}$ is defined on a closed subset of $\bar{\Omega} \times \bar{\Omega} \times[0,1]$. We slightly change our notation as follows.

Definition 9. We say that the triple $\left(y_{1}, y_{2}, \mu\right)$ is in the interior, provided each of $y_{1}, y_{2}, y_{3}$ is in $\Omega$. It is on the boundary if at least one of $y_{1}, y_{2}, y_{3}$ is in $\partial \Omega$.

For a function $u \in C(\bar{\Omega}), \mathscr{C}$ defined on a closed subset of $\bar{\Omega} \times \bar{\Omega} \times[0,1]$, is continuous on its domain. Hence $\mathscr{b}$ does attain its maximum value somewhere. The following lemma is a concavity maximum principle giving a sufficient condition for the positive maximum not to be attainable at interior points.

Lemma 10. Let $\Omega \subset \mathbb{R}^{n}$ be a smooth bounded domain. Suppose $u \in C^{2}(\Omega) \cap C(\bar{\Omega})$ satisfies the elliptic equation

$$
\Delta u=b(x, u, \nabla u) \quad \text { in } \Omega,
$$

where $b$ satisfies $\partial b / \partial u \geq 0, b$ jointly concave with respect to $(x, u)$. Then if $b$ is anywhere positive, it attains its positive maximum on the boundary (Definition 9).

Proof. This is a special case of Theorem 1.3 in [Korevaar 1983].

On the other hand, another concavity maximum principle gives a sufficient condition to that the positive maximum does be attained at the interior points.

Lemma 11. Let $\Omega$ be smooth, strictly convex and bounded. Let $u$ be such that its graph $S_{u}$ has tangent planes $\pi_{x}$, for all $x \in \partial \Omega$. If each of these boundary planes lies beneath $S_{u}$ (contacting it only at $(x, u(x))$ ), then $\mathscr{b}$ does not attain any positive maximum on the boundary (Definition 9).

Proof. This is Lemma 2.1 in [Korevaar 1983].

A combination immediately yields that if a function $u$ satisfies both Lemma 10 and Lemma 11, then $u$ is convex (not strictly convex). One can get more results about the convexity of a function. (See [Korevaar 1983] for more information.) 


\section{Proofs of Theorem 1 and Corollary 2}

In our particular situation (5), we have to show strict convexity for $h / 2-\log f_{1}$. Firstly we investigate the equation it satisfies. Recall that we use the notation $\varphi=-\log f_{1}$ and $\psi=V-\Delta h / 2+|\nabla h(x)|^{2} / 4$.

Lemma 12. We have the following equation for $h / 2+\varphi$ :

$$
\Delta\left(\frac{h}{2}+\varphi\right)=\left|\nabla\left(\frac{h}{2}+\varphi\right)\right|^{2}-\psi+\lambda_{1} .
$$

Proof. A direct calculation shows

$$
\Delta \varphi=-\frac{\Delta f_{1}}{f_{1}}+\frac{\left|\nabla f_{1}\right|^{2}}{f_{1}^{2}}=\nabla h \cdot \nabla \varphi-V+\lambda_{1}+|\nabla \varphi|^{2} .
$$

Notice that

$$
\left|\nabla\left(\frac{h}{2}+\varphi\right)\right|^{2}=\frac{|\nabla h|^{2}}{4}+|\nabla \varphi|^{2}+\nabla h \cdot \nabla \varphi
$$

and thus

$$
|\nabla \varphi|^{2}+\nabla h \cdot \nabla \varphi=\left|\nabla\left(\frac{h}{2}+\varphi\right)\right|^{2}-\frac{|\nabla h|^{2}}{4} .
$$

Substituting (12) into (11), we conclude

$$
\Delta\left(\frac{h}{2}+\varphi\right)=\frac{\Delta h}{2}-V+\lambda_{1}+\left|\nabla\left(\frac{h}{2}+\varphi\right)\right|^{2}-\frac{|\nabla h|^{2}}{4},
$$

which implies the conclusion.

Remark. Though we can try to apply Lemma 10 and Lemma 11 to the function $h / 2+\varphi$, we can only get convexity (not strict convexity) of it. However, we need the strict convexity. Let

$$
\Psi\left(x, \nabla\left(\frac{h}{2}+\varphi\right)\right)=\left|\nabla\left(\frac{h}{2}+\varphi\right)\right|^{2}-\psi(x)+\lambda_{1} .
$$

Equation (10) becomes

$$
\Delta\left(\frac{h}{2}+\varphi\right)=\Psi\left(x, \nabla\left(\frac{h}{2}+\varphi\right)\right) .
$$

Compared with Lemma 10, $\Psi(x, \nabla(h / 2+\varphi))$ does not depend on $h / 2+\varphi$ itself. Luckily, in this case we can obtain strict convexity, provided $\Psi(x, \nabla(h / 2+\varphi))$ is strictly convex with respect to $x$. We derive the following lemma to make this precise.

Lemma 13. Let $\Omega \subset \mathbb{R}^{n}$ be a smooth strictly convex bounded domain. Let $u \in$ $C^{2}(\Omega) \cap C(\bar{\Omega})$ satisfy

$$
\Delta u=|\nabla u|^{2}-\Phi(x) \text { for all } x \in \Omega,
$$


where $\Phi$ is a smooth function in $\Omega$. Let $\xi(x)=u(x)-\frac{1}{2} \sqrt{c / 2} \sum_{i=1}^{n} x_{i}^{2}$, where $c$ is a nonnegative constant. Assume that

(A1) for all $x \in \partial \Omega$, the tangent plane $\pi_{x}$ at $x$ lies beneath the graph $S_{\xi}$, contacting it only at $(x, \xi(x))$, and

(A2) for all $x \in \Omega$ we have $\operatorname{Hess}_{x}(\Phi)-c I \geq 0$.

Then

$$
\operatorname{Hess}_{x} u-\sqrt{\frac{c}{2}} I \geq 0 \quad \text { for all } x \in \Omega .
$$

Proof. We can see that the conclusion equals to that the function $\xi$ is convex. We will show this by applying Lemma 10 and Lemma 11 to function $\xi$.

By direct computation, we have

$$
|\nabla u|^{2}=|\nabla \xi|^{2}+\frac{c}{2} \sum_{i=1}^{n} x_{i}^{2}+2 \sqrt{\frac{c}{2}} \nabla \xi \cdot x \quad \text { and } \quad \Delta u=\Delta \xi+\sqrt{\frac{c}{2}} n .
$$

From these two equations and (13), we obtain

$$
\Delta \xi=|\nabla \xi|^{2}+2 \sqrt{\frac{c}{2}} \nabla \xi \cdot x-\left(\Phi(x)-\frac{c}{2} \sum_{i=1}^{n} x_{i}^{2}\right)-\sqrt{\frac{c}{2}} n=B(x, \nabla \xi)
$$

Since $B$ does not depend on $\xi$ itself, $\partial B / \partial \xi=0$. All we have to check is Hess $_{x} B \geq 0$. A direct computation shows that

$$
\frac{\partial B}{\partial x_{i}}=2 \sqrt{\frac{c}{2}} \xi_{i}-\left(\frac{\partial \Phi}{\partial x_{i}}-c x_{i}\right) \text { and } \frac{\partial^{2} B}{\partial x_{j} \partial x_{i}}=-\left(\frac{\partial^{2} \Phi}{\partial x_{j} \partial x_{i}}-c \delta_{i j}\right),
$$

which implies $\operatorname{Hess}_{x} B=-\left(\operatorname{Hess}_{x}(\Phi)-c I\right)$. Using our assumption $\operatorname{Hess}_{x}(\Phi)-$ $c I \geq 0$, we conclude that $B$ is concave with respect to $x$.

In view of Lemma 10, we know that if the concavity function $\mathscr{b}$ of $\xi$ is anywhere positive, it attains its positive maximum on the boundary (Definition 9). On the other hand, Lemma 11 tells us that $\mathscr{b}$ does not attain any positive maximum on the boundary (Definition 9). So the concavity function $\mathscr{b}$ of $\xi$ is nonpositive, which implies that $\xi$ is convex.

Remark. Noticing that $h / 2-\log f_{1}$ has no definition on $\partial \Omega$, we only can use Lemma 13 on a subset of $\Omega$. Fortunately, if we can show that $h / 2-\log f_{1}$ is uniformly and strictly convex on any subset of $\Omega$, then it is strictly convex on $\Omega$. In order to show this we have to find a positive constant $b$ such that

$$
\frac{h}{2}-\log f_{1}-b \sum_{i=1}^{n} x_{i}
$$

satisfies assumption (A1) in Lemma 13 near the boundary $\partial \Omega$. More generally, we will show it holds for a wide class of smooth transformations: 
Theorem 14. Let $\Omega$ be a smooth bounded strictly convex domain in $\mathbb{R}^{n}$. Let $u \in$ $C^{2}(\bar{\Omega})$ satisfy

$$
u=0 \quad \text { on } \partial \Omega, \quad u>0 \quad \text { in } \Omega, \quad D u \cdot v>0 \quad \text { on } \partial \Omega,
$$

where $v$ is the interior normal to $\partial \Omega$. Let a transformation function $F$ be

$$
F(x, t)=g(x)+f(t), \quad x \in \Omega, t \in \mathbb{R}^{+} .
$$

Assume $g \in C^{2}(\bar{\Omega})$ and assume $f(t) \in C^{2}\left(\mathbb{R}^{+}\right)$satisfies

$$
f^{\prime}<0, \quad \lim _{t \rightarrow 0^{+}} f^{\prime}=-\infty, \quad f^{\prime \prime}>0, \quad \lim _{t \rightarrow 0^{+}} \frac{f^{\prime}}{f^{\prime \prime}}=0, \quad \lim _{t \rightarrow 0^{+}} \frac{f}{f^{\prime}}=0 .
$$

Then, for $\delta>0$ small enough, the function $w(x)=F(x, u(x))$ is such that $\pi_{x}$ lies beneath $S_{w}$ (contacting only at $(x, w(x))$ ), for all $x \in \partial \Omega_{\delta}$, where

$$
\Omega_{\delta}:=\{x \in \Omega \mid d(x, \partial \Omega)>\delta\} .
$$

Remark. This theorem is a generalization of a result in [Korevaar 1983], which deals with the case of a homogeneous transformation function $F$. However, in studying the convexity of the first eigenfunction of problem (1), we have to deal with nonhomogeneous $F$.

Proof. The conclusion equals to that if $\delta$ is small enough, then

$$
A_{x}^{\delta}:=\left\{y \in \Omega_{\delta} \mid S_{w}(y) \text { lies beneath } \pi_{x}(y) \text { or } S_{w}(y)=\pi_{x}(y)\right\}
$$

is an empty set, for all $x \in \partial \boldsymbol{\Omega}_{\delta}$. We will prove this by the following two facts. Fact 1 says when $x$ is near to $\partial \Omega, A_{x}^{\delta}$ is also near $\partial \Omega$. While Fact 2 tells us that we do find a narrow strip between $\partial \Omega$ and $A_{x}^{\delta}$, no matter how small $\delta$ is. Obviously, these two facts are totally incompatible, unless $A_{x}^{\delta}$ is empty.

Fact 1. Given $\varepsilon>0$, the exists $\delta_{0}>0$ such that $A_{x}^{\delta} \cap \Omega_{\varepsilon}=\varnothing$ for all $0<\delta<\delta_{0}$ and all $x \in \partial \Omega_{\delta}$.

Proof. We show this by comparing the height of graph $S_{w}$ with the height of the tangent plane $\pi_{x}$ directly.

Let $y=\left(y_{1}, y_{2}, \ldots, y_{n}\right) \in \Omega$ and let $x=\left(x_{1}, x_{2}, \ldots, x_{n}\right) \in \partial \Omega_{\delta}$. Then the coordinate of the graph of function $w(y)=F(y, u(y))=g(y)+f(u(y))$ is

$$
S_{w}(y)=\left(y_{1}, y_{2}, \ldots, y_{n}, S_{w}^{n+1}(y)\right),
$$

where $S_{w}^{n+1}(y)=g(y)+f(u(y))$. The coordinate of the tangent plane at $x$ is

$$
\pi_{x}(y)=\left(y_{1}, y_{2}, \ldots, y_{n}, \pi_{x}^{n+1}(y)\right) .
$$

One of the normal directions of $\pi_{x}$ is

$$
\mu=\left(D_{x} F(x, u(x)),-1\right)=\left(D_{x} g+f^{\prime}(u(x)) D_{x} u(x),-1\right) .
$$


From the definition of a normal vector, we know

which implies

$$
0=\left(y-x, \pi_{x}^{n+1}(y)-S_{w}^{n+1}(x)\right) \cdot \mu,
$$

$$
\pi_{x}^{n+1}(y)=(y-x) \cdot\left(D_{x} g+f^{\prime}(u(x)) D_{x} u(x)\right)+S_{w}^{n+1}(x) .
$$

Hence,

$$
\begin{aligned}
& S_{w}^{n+1}(y)-\pi_{x}^{n+1}(y) \\
& \quad=S_{w}^{n+1}(y)-(y-x) \cdot\left(D_{x} g+f^{\prime}(u(x)) D_{x} u(x)\right)-S_{w}^{n+1}(x) \\
& \quad=g(y)+f(u(y))-g(x)-f(u(x))-(y-x) \cdot D_{x} g-f^{\prime}(u(x))(y-x) \cdot D_{x} u(x) \\
& =f^{\prime}(u(x))\left(\frac{Q(x, y)}{f^{\prime}(u(x))}-\frac{f(u(x))}{f^{\prime}(u(x))}-(y-x) \cdot D_{x} u(x)\right),
\end{aligned}
$$

where

$$
Q(x, y):=g(y)+f(u(y))-g(x)-(y-x) \cdot D_{x} g .
$$

Notice that $Q(x, y)$ is bounded on $\Omega \times \Omega_{\varepsilon}$, since $g \in C^{1}(\bar{\Omega}), f \in C^{2}\left(\mathbb{R}^{+}\right)$and $\Omega$ is bounded by assumption. That is to say, we can choose a positive constant $C_{1}>0$ such that

$$
|Q(x, y)|<C_{1} \quad \text { for all }(x, y) \in \Omega \times \Omega_{\varepsilon} .
$$

Extending the normal vector field $v$ smoothly in a neighborhood of $\partial \Omega$, we can talk about normal directions in the entire neighborhood. Since $\partial \Omega$ is a level set of $u$ by (15), $D u(x)$ is a positive multiple of the interior normal $v(x)$, for $x \in \partial \Omega$. So when $\delta$ is small enough, $D u(x)$ is close to $v(x)$ for $x \in \partial \Omega_{\delta}$. Hence, we can choose $\delta_{1}>0$ small enough and a positive constant $C_{2}$ such that

$$
(y-x) \cdot D u(x)>C_{2}>0 \quad \text { for all } y \in \Omega_{\varepsilon} \text { and } x \in \Omega \backslash \Omega_{\delta_{1}} .
$$

We have used the strict convexity of $\Omega$ and the compactness of $\partial \Omega$ to gain estimate (18).

From (17) and the assumptions $\lim _{t \rightarrow 0^{+}} f^{\prime}=-\infty$ and $\lim _{t \rightarrow 0^{+}} f / f^{\prime}=0$ in (16), we can choose a positive $\delta_{2}<\delta_{1}$ such that

$$
\left|\frac{f(u(x))}{f^{\prime}(u(x))}\right|<\frac{1}{4} C_{2} \text { and }\left|\frac{Q(x, y)}{f^{\prime}(u(x))}\right|<\frac{1}{4} C_{2} \quad \text { for all } y \in \Omega_{\varepsilon} \text { and } x \in \Omega \backslash \Omega_{\delta_{2}} .
$$

From (18) (19) and the assumption $f^{\prime}<0$, we have

$$
S_{w}^{n+1}(y)-\pi_{x}^{n+1}(y)>-\frac{C_{1}}{2} f^{\prime}(u(x))>0 \quad \text { for all } y \in \Omega_{\varepsilon} \text { for all } x \in \Omega \backslash \Omega_{\delta_{2}},
$$

which implies $A_{x}^{\delta} \cap \Omega_{\varepsilon}=\varnothing$, for all $x \in \partial \Omega_{\delta}, 0<\delta<\delta_{2}$.

We now show that $w$ is convex in a boundary strip about $\partial \Omega$. 
Fact 2. There exists $\varepsilon>0$ such that $\operatorname{Hess}(w(x))>0$ for all $x \in \Omega \backslash \Omega_{\varepsilon}$.

Proof. To show this, we study the terms comprising

$$
\operatorname{Hess}(w)=\operatorname{Hess}(g)+f^{\prime \prime}(u)\left(D_{x} u\right)\left(D_{x} u\right)^{t}+f^{\prime}(u) \operatorname{Hess}(u) .
$$

As in the proof of Fact 1, we extend the normal vector field $v(x)$ smoothly into a strip about $\partial \Omega$ and then we can continue to talk about tangential directions $(v(x)$. $\eta=0)$ and nontangential ones.

Let $\eta(x)=\left(\eta_{1}(x), \eta_{2}(x), \ldots, \eta_{n}(x)\right)$ be a vector at point $x$. The conclusion equals to $\eta(x) \operatorname{Hess}(w(x)) \eta^{t}(x)>0$, for all $\eta(x) \neq 0$, for all $x \in \Omega \backslash \Omega_{\varepsilon}$. Actually, we only have to show this for a set of orthonormal basis. When $\varepsilon$ is sufficiently small, we can choose a set of smooth vector field $\left\{e_{1}(x), e_{2}(x), \ldots, e_{n}(x)\right\}$, such that $\left\{e_{1}(x), e_{2}(x), \ldots, e_{n}(x)\right\}$ is an orthonormal basis at $x \in \Omega \backslash \Omega_{\varepsilon}, e_{1}(x)$ is close to $v(x)$ and each $e_{i}(x)(i \neq 1)$ is close to some tangential direction. Moreover, since the boundary $\partial \Omega$ is compact and $D u(x)$ is a positive multiple of the interior normal $v$ when $x \in \partial \Omega$, we can assume that for any $\frac{1}{2}>a>0$ there exists $\varepsilon_{1}>0$ such that

$$
\begin{aligned}
\left|e_{i}(x) \cdot D u(x)\right|<a & & \text { for all } x \in \Omega \backslash \Omega_{\varepsilon_{1}} \text { and } i \neq 1, \\
e_{1}(x) \cdot D u(x)>1-a & & \text { for all } x \in \Omega \backslash \Omega_{\varepsilon_{1}} .
\end{aligned}
$$

For $\eta=e_{1}$, which is close to the normal direction, we have

$$
\begin{aligned}
\eta \operatorname{Hess}(w) \eta^{t} & =\eta \operatorname{Hess}(g) \eta^{t}+f^{\prime \prime}(u) \eta\left(D_{x} u\right)\left(D_{x} u\right)^{t} \eta^{t}+f^{\prime}(u) \eta \operatorname{Hess}(u) \eta^{t} \\
& =f^{\prime \prime}(u)\left(P(x)+\eta\left(D_{x} u\right)\left(D_{x} u\right)^{t} \eta^{t}\right)
\end{aligned}
$$

where

$$
P(x):=\frac{\eta \operatorname{Hess}(g) \eta^{t}}{f^{\prime \prime}(u)}+\frac{f^{\prime}(u)}{f^{\prime \prime}(u)} \eta \operatorname{Hess}(u) \eta^{t} .
$$

From the assumptions $f^{\prime \prime}>0, \lim _{t \rightarrow 0^{+}} f^{\prime}=-\infty$ and $\lim _{t \rightarrow 0^{+}} f^{\prime} / f^{\prime \prime}=0$ in (16), we have

$$
\lim _{t \rightarrow 0^{+}} f^{\prime \prime}(t)=+\infty
$$

By the continuity of $u_{i j}$ and $g$ on $\bar{\Omega}$, combined with (22) and the assumption that $\lim _{t \rightarrow 0^{+}} f^{\prime} / f^{\prime \prime}=0$, there exists a positive $\varepsilon_{2}<\varepsilon_{1}$ such that

$$
|P(x)|<\frac{1}{2}(1-a)^{2} \quad \text { for all } x \in \Omega \backslash \Omega_{\varepsilon_{2}} .
$$

Therefore, using (20) and assumption that $f^{\prime \prime}>0$, we have

$$
\eta \operatorname{Hess}(w) \eta^{t}>f^{\prime \prime}(u)\left(-\frac{1}{2}(1-a)^{2}+(1-a)^{2}\right)>0 \quad \text { for all } x \in \Omega \backslash \Omega_{\varepsilon_{2}} .
$$


As to $\eta=e_{i}(i \neq 1)$, which is close to the tangential direction,

$$
\begin{aligned}
\eta \operatorname{Hess}(w) \eta^{t} & =\eta \operatorname{Hess}(g) \eta^{t}+f^{\prime \prime}(u) \eta\left(D_{x} u\right)\left(D_{x} u\right)^{t} \eta^{t}+f^{\prime}(u) \eta \operatorname{Hess}(u) \eta^{t} \\
& \geq \eta \operatorname{Hess}(g) \eta^{t}+f^{\prime}(u) \eta \operatorname{Hess}(u) \eta^{t}
\end{aligned}
$$

We have used the positivity of $f^{\prime \prime}$ and positive semidefiniteness of the matrix $\left(D_{x} u\right)\left(D_{x} u\right)^{t}$ to gain $(23)$.

If $x \in \partial \Omega$, the matrix Hess $u(x)$ is negative definite in all tangential directions, that is, there exists a positive constant $k>0$ such that $\eta \operatorname{Hess}(u) \eta<-k|\eta|^{2}=-k$ for any tangential direction $\eta$. From the compactness of $\partial \Omega$ and the assumption $u \in C^{2}(\bar{\Omega})$, there exists a positive $\varepsilon_{3}<\varepsilon_{2}$ such that

$$
\eta(x) \operatorname{Hess} u(x) \eta^{t}(x)<-k, \text { for all } x \in \Omega \backslash \Omega \varepsilon_{3} .
$$

From the continuity of $g_{i j}$ on $\bar{\Omega}$ and the assumption that $\lim _{t \rightarrow 0^{+}} f^{\prime}=-\infty$, we can choose a positive $\varepsilon_{4}<\varepsilon_{3}$ such that

$$
\frac{\eta \operatorname{Hess}(g) \eta^{t}}{-f^{\prime}(u)}>-\frac{1}{2} k>\eta(x) \operatorname{Hess} u(x) \eta^{t}(x), \text { for all } x \in \Omega \backslash \Omega \varepsilon_{4} \text {. }
$$

Combining (23) (24) and (25), we have for all $x \in \Omega \backslash \Omega \varepsilon_{4}$

$$
\eta \operatorname{Hess}(w) \eta^{t} \geq-f^{\prime}(u)\left(\frac{\eta \operatorname{Hess}(g) \eta^{t}}{-f^{\prime}(u)}-\eta \operatorname{Hess}(u) \eta^{t}\right)>-\frac{1}{2} k f^{\prime}(u)>0 .
$$

In conclusion, if $\varepsilon<\varepsilon_{4}$, then $\eta^{t}(x) \operatorname{Hess}(w)(x) \eta(x)>0$ for all $x \in \Omega \backslash \Omega \varepsilon_{4}$ and for all $\eta(x) \neq 0$, which implies Fact 2.

Theorem 14 now follows from Fact 1 and Fact 2 together: Pick $\varepsilon>0$ such that $\operatorname{Hess}(w)(x)>0$ for $x \in \Omega \backslash \Omega_{\varepsilon}$. For this $\varepsilon>0$, pick $\delta_{0}$ so that for $0<\delta<\delta_{0}$ and $x \in \partial \boldsymbol{\Omega}_{\delta}$, we have $A_{x} \cap \boldsymbol{\Omega}_{\varepsilon}=\varnothing$. Because $\operatorname{Hess}(w)(x)>0$ in $\Omega \backslash \boldsymbol{\Omega}_{\varepsilon}$, we also have $A_{x} \cap\left(\Omega_{\delta} \backslash \Omega_{\varepsilon}\right)=\varnothing$. Hence for $0<\delta<\delta_{0}, A_{x}=\varnothing$, which implies for small enough $\delta$, tangent planes $\pi_{x}$ lies beneath $S_{w}$ for all $x \in \partial \Omega_{\delta}$.

Proof of Theorem 1. Recall that in Lemma 12 we have shown

$$
\Delta\left(\frac{h}{2}+\varphi\right)=\left|\nabla\left(\frac{h}{2}+\varphi\right)\right|^{2}-\psi(x)+\lambda_{1},
$$

where $\varphi=-\log f_{1}$ and $\psi=V-\frac{1}{2} \Delta h+\frac{1}{4}|\nabla h|^{2}$.

First we will show for small enough $\delta>0, \xi=\frac{1}{2} h+\varphi-\frac{1}{2} \sqrt{c / 2} \sum_{i=1}^{n} x_{i}$ satisfies assumption (A1) in Lemma 13: for all $x \in \partial \Omega, \pi_{x}$ lies beneath $S_{\xi}$, contacting it only at $(x, \xi(x))$.

Choosing the transformation function $F(x, t)=g(x)+f(t)$, where

$$
g(x)=\frac{h(x)}{2}-\frac{1}{2} \sqrt{\frac{c}{2}} \sum_{i=1}^{n} x_{i} \quad \text { and } \quad f(t)=-\log t,
$$


we can write

$$
\xi=\frac{h}{2}+\varphi-\frac{1}{2} \sqrt{\frac{c}{2}} \sum_{i=1}^{n} x_{i}=F\left(x, f_{1}(x)\right) .
$$

Thus, using Theorem 14 we see that $\pi_{x}$ lies beneath $S_{\xi}$ for all $x \in \partial \Omega_{\delta}$ with $\delta>0$ small enough.

Let $\Phi=\psi-\lambda_{1}$. Since Hess $x \psi-c I \geq 0$ for all $x \in \Omega$, we have $\operatorname{Hess}_{x} \Phi=$ Hess $_{x} \psi \geq c I$ for all $x \in \Omega$. Therefore, for $\delta>0$ small enough, $h / 2+\varphi$ satisfies Lemma 13 in the domain $\Omega_{\delta}$. Since $\Omega$ is strictly convex, we can still assume $\Omega_{\delta}$ is strictly convex. By using Lemma 13 on $\Omega_{\delta}$, we get

$$
\operatorname{Hess}_{x}\left(\frac{h}{2}+\varphi\right)-\sqrt{\frac{c}{2}} I \geq 0 \quad \text { in } \Omega_{\delta} .
$$

Since $\delta$ can be any sufficiently small positive constant, (26) is also valid in $\Omega$.

Proof of Corollary 2. Recall from Equation (5) that

$$
\Delta u=-\lambda u+2 \nabla u \cdot \nabla\left(\frac{h}{2}-\log f_{1}\right)
$$

We already know that $h / 2+\varphi$ is strictly convex and that $u$ satisfies the Neumann boundary condition $\partial u / \partial v=0$ (Lemma 6). Combining Lemma 7 and Theorem 1 , we obtain the estimate (2).

\section{Proofs of Theorem 3 and Corollary 4}

Equation (5) will satisfies the hypothesis of Lemma 7 if

$$
\operatorname{Hess}_{x}\left(\frac{h}{2}+\varphi\right)-\sqrt{\frac{c}{2}} I \geq 0,
$$

otherwise we can still obtain the following estimate.

Lemma 15. Let $\Omega \subset \mathbb{R}^{n}$ be a smooth and bounded domain. Let $\tau_{i}(x)(i=1, \ldots, n)$ be the eigenvalues of $\operatorname{Hess}_{x} \varphi$ at the point $x$ and let $\kappa_{i}(x)(i=1, \ldots, n)$ be the eigenvalues of $\operatorname{Hess}_{x}(h / 2+\varphi)$ at $x$. Then

$$
\min _{1 \leq i \leq n} \inf _{x \in \Omega} \tau_{i}(x)>-\infty
$$

equivalently, there exists a constant $a \geq 0$ such that

$$
\min _{1 \leq i \leq n} \inf _{x \in \Omega} \tau_{i}(x) \geq-a .
$$

Since $h$ is smooth, the same holds for $\min _{1 \leq i \leq n} \inf _{x \in \Omega} \kappa_{i}(x)$.

Proof. The conclusion is equivalent to the existence of a constant $a \geq 0$ such that Hess $\varphi(x)+a I \geq 0$ for all $x \in \Omega$. We find the constant by computing the Hessian 
of $\varphi$ directly. Since $\varphi$ is smooth in $\Omega$, we only need to study what happens when $x$ is near to the boundary.

For any $p \in \partial \Omega$, we choose the same local coordinates $\left\{x_{1}, x_{2}, \ldots, x_{n}\right\}$ and the neighborhood $U$ as in Lemma 6. Similar as in there we can write locally $f_{1}=x_{1} \cdot g$. Recall that $g$ is a smooth function and $g \neq 0$ in $\bar{\Omega} \cap U$.

Then locally we have

$$
\varphi_{i}=-\frac{\left(f_{1}\right)_{i}}{f_{1}}=-\frac{\left(x_{1} g\right)_{i}}{x_{1} g}
$$

When $i=1$, we have

$$
\varphi_{11}=-\frac{\left(f_{1}\right)_{11}}{f_{1}}+\frac{\left(f_{1}\right)_{1}^{2}}{f_{1}^{2}},
$$

from which we can see that

$$
f_{1}^{2} \varphi_{11}=-\left(f_{1}\right)_{11} f_{1}+\left(f_{1}\right)_{1}^{2} .
$$

Since $f_{1}$ is smooth up to the boundary and $f_{1}=0$ on $\partial \Omega$. The Hopf lemma shows that $\partial f_{1} / \partial x_{1} \neq 0$ on $\partial \Omega$. So the right-hand side of (27) tends to a finite positive number as $x \rightarrow p \in \partial \Omega$. Therefore

$$
\lim _{x \rightarrow p} \varphi_{11}=+\infty
$$

For $2 \leq i \leq n$, we have

$$
\varphi_{i}=-\frac{g_{i}}{g}
$$

For $1 \leq j \leq n$, we have

$$
\varphi_{i j}=-\frac{g_{i j}}{g}+\frac{g_{i} g_{j}}{g^{2}}
$$

which tends to finite value as $x \rightarrow p \in \partial \Omega$. In conclusion, $\varphi_{11} \rightarrow+\infty$ as $x \rightarrow p$ and $\varphi_{i j}(i \neq 1$ or $j \neq 1)$ tend to finite numbers as $x \rightarrow p$. So for any small neighborhood $V$ of $p$, we can choose a sufficiently large $a$ such that

$$
\operatorname{Hess} \varphi(x)+a I \geq 0 \quad \text { for all } x \in V .
$$

Since $\Omega$ is a bounded domain and $\varphi$ is smooth in $\Omega$, there exists an uniform number $a$ such that

$$
\text { Hess } \varphi(x)+a I \geq 0 \quad \text { for all } x \in \Omega .
$$

Thus, we obtain the conclusion.

In view of Lemma 15, we will assume

$$
\min _{1 \leq i \leq n} \inf _{x} \kappa_{i} \geq-a,
$$

where $a$ is a nonnegative constant. 
Proof of Theorem 3. Following [Yau 2003], we consider the function

$$
F(x)=\frac{|\nabla u(x)|^{2}}{(c-u(x))^{2}}+\alpha \log (c-u(x)),
$$

for $c>\sup _{x} u$ and $\alpha>0$ as selected below. Actually, we try to find those constants $\alpha$ and $c$ such that $|\nabla u|=0$ at the maximum points of $F$.

By some computations, we have

$$
\begin{aligned}
& F_{i}=2 \sum_{j=1}^{n} u_{j} u_{j i}(c-u)^{-2}+2|\nabla u|^{2}(c-u)^{-3} u_{i}-\alpha(c-u)^{-1} u_{i} \\
& \Delta F=2\left|D^{2} u\right|^{2}(c-u)^{-2}+2(\nabla u \cdot \nabla \Delta u)(c-u)^{-2}+6(c-u)^{-4}|\nabla u|^{4} \\
& +2(c-u)^{-3}(\Delta u)|\nabla u|^{2}+8 \sum_{i, j=1}^{n} u_{j} u_{j i} u_{i}(c-u)^{-3} \\
& -\alpha|\nabla u|^{2}(c-u)^{-2}-\alpha(c-u)^{-1} \Delta u .
\end{aligned}
$$

Case 1. Suppose $F$ attains its maximum on $\partial \Omega$ at a point $x_{0}$. We can choose an orthonormal frame $\left\{l_{1}, l_{2}, \ldots, l_{n}\right\}$ around $x_{0}$ such that $l_{n}$ is perpendicular to $\partial \Omega$ and pointing outward. We also use the notation $\partial / \partial x_{n}$ to denote the restriction of $l_{n}$ on $\partial \Omega$.

A computation shows that, at the maximum point $x_{0} \in \partial \Omega$,

$$
\begin{aligned}
0 \leq \frac{\partial F}{\partial x_{n}}\left(x_{0}\right) & =2 \sum_{j=1}^{n-1} u_{j} u_{j n}(c-u)^{-2}+2|\nabla u|^{2}(c-u)^{-3} u_{n}-\alpha(c-u)^{-1} u_{n} \\
& =2 \sum_{j=1}^{n-1} u_{j} u_{j n}(c-u)^{-2} .
\end{aligned}
$$

We have used that $\left(\partial u / \partial x_{n}\right)(x)=0$ for $x \in \partial \Omega$ (see Lemma 6). From the definition of the second fundamental form of a hypersurface in $\mathbb{R}^{n}$, we have

$$
u_{j n}=-\sum_{k=1}^{n-1} h_{j k} u_{k} \quad \text { for all } 1 \leq j \leq n-1,
$$

where $h_{j k}$ is the second fundamental form of $\partial \Omega$. Therefore we obtain

$$
0 \leq \frac{\partial F}{\partial x_{n}}=-2 \sum_{j, k=1}^{n-1} u_{j} h_{j k} u_{k}(c-u)^{-2} \leq 0 .
$$

We have used the positivity of $h_{j k}$, arising from the assumption that $\partial \Omega$ is strictly convex. Therefore, $|\nabla u|=0$ at $x_{0}$. 
Thus for all $x \in \bar{\Omega}$, we have

$$
F(x) \leq F\left(x_{0}\right)=\alpha \log \left(c-u\left(x_{0}\right)\right) \leq \alpha \log c .
$$

Case 2. Suppose that $F$ attains its maximum in an interior point $x_{0}$ of $\Omega$ and that $\nabla u\left(x_{0}\right)=0$. In this case, we still can get (32).

Case 3. Suppose that $F$ attains its maximum in an interior point $x_{0}$ of $\Omega$ and that $\nabla u\left(x_{0}\right) \neq 0$.

In this case, we can choose a coordinate so that

$$
u_{1}\left(x_{0}\right) \neq 0, \quad u_{i}\left(x_{0}\right)=0,2 \leq i \leq n .
$$

Using (33) we can rewrite (30) as

$$
F_{i}\left(x_{0}\right)=2 u_{1} u_{1 i}(c-u)^{-2}+2 u_{1}^{2}(c-u)^{-3} u_{i}-\alpha(c-u)^{-1} u_{i} .
$$

Since $F_{1}\left(x_{0}\right)=0$, we get

$$
u_{11}(c-u)^{-1}+u_{1}^{2}(c-u)^{-2}=\frac{\alpha}{2},
$$

from which we can see that

$$
u_{11}=\left(\frac{\alpha}{2}-u_{1}^{2}(c-u)^{-2}\right)(c-u) .
$$

Thus, we have

$$
2\left|D^{2} u\right|^{2}(c-u)^{-2} \geq 2 u_{11}(c-u)^{-2}=\frac{\alpha^{2}}{2}-2 \alpha u_{1}^{2}(c-u)^{-2}+2 u_{1}^{4}(c-u)^{-4} .
$$

We can estimate the second term in the right-hand side of Equation (31) as follows:

$$
\begin{aligned}
2 \nabla u \cdot \nabla(\Delta u)(c-u)^{-2}= & 2 \nabla u \cdot \nabla\left(-\lambda u+2 \nabla\left(\frac{h}{2}+\varphi\right) \cdot \nabla u\right)(c-u)^{-2} \\
= & -2 \lambda|\nabla u|^{2}(c-u)^{-2}+4 u_{i}\left(\frac{h}{2}+\varphi\right)_{j i} u_{j}(c-u)^{-2} \\
& +4 u_{i}\left(\frac{h}{2}+\varphi\right)_{i} u_{i j}(c-u)^{-2} \\
\geq-2 \lambda|\nabla u|^{2}(c-u)^{-2} & +4 u_{i}\left(\frac{h}{2}+\varphi\right)_{i} u_{i j}(c-u)^{-2} \\
& +4|\nabla u|^{2} \min _{i} \inf _{x} \kappa_{i}(x)(c-u)^{-2} .
\end{aligned}
$$

By computation, we obtain

$$
\begin{aligned}
2|\nabla u|^{2}(\Delta u) & (c-u)^{-3} \\
& =2|\nabla u|^{2}\left(-\lambda u+2 \nabla\left(\frac{h}{2}+\varphi\right) \cdot \nabla u\right)(c-u)^{-3} \\
& =-2 \lambda u|\nabla u|^{2}(c-u)^{-3}+4|\nabla u|^{2}\left(\nabla\left(\frac{h}{2}+\varphi\right) \cdot \nabla u\right)(c-u)^{-3}
\end{aligned}
$$


At the maximum point $x_{0}$, we have

$$
\begin{aligned}
& 0=\nabla F \cdot \nabla\left(\frac{h}{2}+\varphi\right) \\
&=2 u_{j} u_{j i}\left(\frac{h}{2}+\varphi\right)_{i}(c-u)^{-2}+2|\nabla u|^{2}(c-u)^{-3} \nabla\left(\frac{h}{2}+\varphi\right) \cdot \nabla u \\
&-\alpha(c-u)^{-1} \nabla\left(\frac{h}{2}+\varphi\right) \cdot \nabla u .
\end{aligned}
$$

We substitute (35), (36) and (37) into (31) and obtain

$$
\begin{aligned}
\Delta F\left(x_{0}\right) \geq \frac{\alpha^{2}}{2}-2 \alpha u_{1}^{2}( & c-u)^{-2}+2 u_{1}^{4}(c-u)^{-4} \\
& -2 \lambda|\nabla u|^{2}(c-u)^{-2}+4|\nabla u|^{2} \min _{i} \inf _{x} \kappa_{i}(c-u)^{-2} \\
& +6 u_{1}^{4}(c-u)^{-4}-2 \lambda u|\nabla u|^{2}(c-u)^{-3} \\
& +8 u_{j} u_{j i} u_{i}(c-u)^{-3}-\alpha|\nabla u|^{2}(c-u)^{-2}+\alpha(c-u)^{-1} \lambda u .
\end{aligned}
$$

By using (34), we can compute that

$$
\begin{aligned}
8|\nabla u|^{4}(c-u)^{-4}+8 u_{j} u_{j i} u_{i}(c-u)^{-3} & =8 u_{1}^{4}(c-u)^{-4}+8 u_{1} u_{11} u_{1}(c-u)^{-3} \\
& =8(c-u)^{-2} u_{1}^{2}\left((c-u)^{-2} u_{1}^{2}+u_{11} u_{1}^{-1}\right) \\
& =4 \alpha(c-u)^{-2}|\nabla u|^{2} .
\end{aligned}
$$

Therefore,

$$
\begin{aligned}
0 \geq & \Delta F\left(x_{0}\right) \\
\geq & \frac{1}{2} \alpha^{2}+\alpha(c-u)^{-2}|\nabla u|^{2}-2 \lambda|\nabla u|^{2}(c-u)^{-2} \\
& \quad-2 \lambda u|\nabla u|^{2}(c-u)^{-3}+\alpha \lambda(c-u)^{-1} u+4|\nabla u|^{2} \min _{i} \inf _{x} \kappa_{i}(x)(c-u)^{-2} \\
\geq & \frac{1}{2} \alpha^{2}+(c-u)^{-2}|\nabla u|^{2}\left(\alpha-2 \lambda-2 \lambda u(c-u)^{-1}+4 \min _{i} \inf _{x} \kappa_{i}(x)\right) \\
\geq & \frac{1}{2} \alpha^{2}+(c-u)^{-2}|\nabla u|^{2}\left(\alpha-2 \lambda-2 \lambda \sup _{x} u\left(c-\sup _{x} u\right)^{-1}+4 \min _{i} \inf _{x} \kappa_{i}(x)\right) .
\end{aligned}
$$

Choosing $c=(1+\varepsilon) \sup _{x} u$ and $\alpha=2 \lambda\left(1+\varepsilon^{-1}\right)-4 \min _{i} \inf _{x \in \Omega} \kappa_{i}(x)$, we get $\Delta F\left(x_{0}\right)>0$, which is a contradiction. Therefore, $\nabla u\left(x_{0}\right)=0$, which means (32) is valid in this case as well.

Our argument above shows that (32) is valid in all cases. A simple computation shows (3).

At last we shall derive our lower bound

$$
2(\operatorname{diam} \Omega)^{-2} \exp \left(-a(\operatorname{diam} \Omega)^{2}-1\right) \leq \lambda_{2}-\lambda_{1} .
$$

Proof of Corollary 4. From (3) we have, for all $\varepsilon>0$,

$$
\left|\nabla \sqrt{\log \frac{c}{c-u}}\right| \leq \frac{1}{2} \sqrt{\alpha}
$$

where $c=(1+\varepsilon) \sup _{x} u$ and $\alpha=2 \lambda\left(1+\varepsilon^{-1}\right)-4 \min _{i} \inf _{x \in \Omega}(h / 2+\varphi)_{i i}$. 
Let $q_{1}, q_{2}$ be two points of $\bar{\Omega}$ such that $u\left(q_{1}\right)=\sup _{x} u, u\left(q_{2}\right)=0$ and $\gamma$ is the line segment joining them. Since $\Omega$ is convex by assumption, $\gamma$ lies in $\Omega$. By integrating both sides of inequality (38) along $\gamma$ from $q_{1}$ to $q_{2}$, we have

$$
\int_{\sup _{x} u}^{0}\left|\frac{d(\log (c /(c-u)))^{1 / 2}}{d u} d u\right| \leq \int_{q_{1}}^{q_{2}} \frac{1}{2} \sqrt{\alpha} d s \leq \frac{1}{2} \sqrt{\alpha}(\operatorname{diam} \Omega) .
$$

By elementary calculus, we have

$$
\left(\log \frac{c}{c-\sup _{x} u}\right)^{1 / 2} \leq \frac{1}{2} \sqrt{\alpha}(\operatorname{diam} \Omega)
$$

which implies

$$
\alpha \geq 4(\operatorname{diam} \Omega)^{-2} \log (1+1 / \varepsilon) .
$$

Putting $\alpha=2 \lambda\left(1+\varepsilon^{-1}\right)-4 \min _{i} \inf _{x \in \Omega} \kappa_{i}(x)$ into (39), and defining $\varepsilon^{\prime}=1+1 / \varepsilon$, we obtain

$$
\begin{aligned}
\lambda_{2}-\lambda_{1} & \geq \varepsilon^{\prime-1}\left(2(\operatorname{diam} \Omega)^{-2} \log \varepsilon^{\prime}+2 \min _{i} \inf _{x} \kappa_{i}(x)\right) \\
& =2(\operatorname{diam} \Omega)^{-2} \varepsilon^{\prime-1} \log \left(\varepsilon^{\prime} \exp \left((\operatorname{diam} \Omega)^{2} \min _{i} \inf _{x} \kappa_{i}(x)\right)\right) .
\end{aligned}
$$

Since $\varepsilon$ can be any positive number and the right-hand side of the preceding equation is at most $2(\operatorname{diam} \Omega)^{-2} \exp \left(\min _{i} \inf _{x} \kappa_{i}(x)(\operatorname{diam} \Omega)^{2}-1\right)$, we obtain

$$
\lambda_{2}-\lambda_{1} \geq 2(\operatorname{diam} \Omega)^{-2} \exp \left(\min _{i} \inf _{x} \kappa_{i}(x)(\operatorname{diam} \Omega)^{2}-1\right) .
$$

Therefore, if $\min _{i} \inf _{x} \kappa_{i}(x) \geq-a$, then

$$
\lambda_{2}-\lambda_{1} \geq 2(\operatorname{diam} \Omega)^{-2} \exp \left(-a(\operatorname{diam} \Omega)^{2}-1\right) .
$$

\section{References}

[Da Prato and Lunardi 2004] G. Da Prato and A. Lunardi, "Elliptic operators with unbounded drift coefficients and Neumann boundary condition", J. Differential Equations 198:1 (2004), 35-52. MR 2004k:35066 Zbl 1046.35025

[González and Negrin 1999] B. J. González and E. R. Negrin, "Gradient estimates for positive solutions of the Laplacian with drift", Proc. Amer. Math. Soc. 127:2 (1999), 619-625. MR 99c:58150 Zbl 0940.58016

[Kawohl 1985] B. Kawohl, Rearrangements and convexity of level sets in PDE, Lecture Notes in Math. 1150, Springer, Berlin, 1985. MR 87a:35001 Zbl 0593.35002

[Korevaar 1983] N. J. Korevaar, "Convex solutions to nonlinear elliptic and parabolic boundary value problems", Indiana Univ. Math. J. 32:4 (1983), 603-614. MR 85c:35026 Zbl 0481.35024

[Li and Wang 2005] P. Li and J. Wang, "Comparison theorem for Kähler manifolds and positivity of spectrum”, J. Differential Geom. 69:1 (2005), 43-74. MR 2006g:53111 Zbl 1087.53067

[Li and Yau 1986] P. Li and S.-T. Yau, "On the parabolic kernel of the Schrödinger operator", Acta Math. 156:3-4 (1986), 153-201. MR 87f:58156 Zbl 0611.58045 
[Ma and Liu 2008] L. Ma and B. Liu, "Convexity of first eigenfunction of drifting Laplacian operator and its application", New York J. Math. 14 (2008), 393-401. Zbl 05321472

[Ma and Zhu 2007] L. Ma and A. Zhu, "Eigenvalues and lambda constants on Riemannian submersions", Geom. Dedicata 129 (2007), 73-82. MR 2008j:53062 Zbl 05228588

[Ni 2004] L. Ni, “The entropy formula for linear heat equation”, J. Geom. Anal. 14:1 (2004), 87100. MR 2004m:53118a Zbl 1044.58030

[Schoen and Yau 1994] R. Schoen and S.-T. Yau, Lectures on differential geometry, Conf. Proc. Lecture Notes in Geom. Topol. I, Int. Press, Cambridge, MA, 1994. Lecture notes prepared by W. Y. Ding, K. C. Chang, J. Q. Zhong and Y. C. Xu, Translated from the Chinese by W. Y. Ding and S. Y. Cheng, Preface translated from the Chinese by K. Tso. MR 97d:53001 Zbl 0830.53001

[Setti 1993] A. G. Setti, "Eigenvalue estimates for the Laplacian with lower order terms on a compact Riemannian manifold”, pp. 521-527 in Differential geometry: Riemannian geometry (Los Angeles 1990), edited by R. Greene and S.-T. Yau, Proc. Sympos. Pure Math. 54, Amer. Math. Soc., Providence, RI, 1993. MR 1216643 Zbl 0788.58056

[Yau 2003] S.-T. Yau, "An estimate of the gap of the first two eigenvalues in the Schrödinger operator", pp. 223-235 in Lectures on partial differential equations, edited by S.-Y. A. Chang et al., New Stud. Adv. Math. 2, Int. Press, Somerville, MA, 2003. MR 2005c:35219 Zbl 1050.35057

Received June 2, 2008. Revised June 4, 2008.

LI MA

DEPARTMENT OF MATHEMATical SCIENCES

TSINGHUA UNIVERSITY

HAIDIAN

BEIJING 100084

CHINA

lma@math.tsinghua.edu.cn

BAIYU LIU

Department of MATHEMATiCAl SCIEnCES

TSINGHUA UNIVERSITY

HAIDIAN

BEIJING 100084

ChINA

liuby05@mails.tsinghua.edu.cn 